\title{
Vibration of Nano Beam Induced by Ramp Type Heating
}

\author{
Hamdy M. Youssef ${ }^{1}$, Khaled A. Elsibai ${ }^{2}$ \\ ${ }^{1}$ Faculty of Engineering, Umm Al-Qura University, Makkah, Saudi Arabia \\ ${ }^{2}$ Mathematics Department, Faculty Science, Umm Al-Qura University, Makkah, Saudi Arabia \\ E-mail: \{yousefanne, drkhaledelsibai\}@yahoo.com \\ Received March 23, 2011; revised April 18, 2011; accepted May 8, 2011
}

\begin{abstract}
The non-Fourier effect in heat conduction and the coupling effect between temperature and strain rate, became the most significant effects in the nano-scale beam. In the present study, a generalized solution for the generalized thermoelastic vibration of a bounded nano-beam resonator induced by ramp type of heating is developed and the solutions take into account the above two effects. The Laplace transforms and direct method are used to determine the lateral vibration, the temperature, the displacement, the stress and the energy of the beam. The effects of the relaxation time and the ramping time parameters have been studied with some comparisons.
\end{abstract}

Keywords: Thermoelasticity, Nano-Beam, Ramp-Type Heating, Non-Fourier Heat Conduction

\section{Introduction}

The generalized thermoelasticity theories have been developed with the aim of removing the paradox of infinite speed of heat propagation inherent in the classical dynamical coupled thermoelasticity theory (Biot-CTE) [1], Lord and Shulman (L-S) [2] obtained a wave-type heat equation by postulating a new law of heat conduction to replace the classical Fourier's law. Since the heat equation of this theory is of the wave-type, it automatically ensures finite speeds of propagation for heat and elastic waves. The remaining governing equations for this theory, namely, the equations of motion and constitutive relations, remain the same as those for the coupled theory.

Many attempts have been made recently to investigate the elastic properties of nanostructured materials by atomistic simulations. Diao et al. [3] studied the effect of free surfaces on the structure and elastic properties of gold nanowires by atomistic simulations. Although the atomistic simulation is a good way to calculate the elastic constants of nanostructured materials, it is only applicable to homogeneous nanostructured materials (e.g., nanoplates, nanobeams, nanowires, etc.) with limited number of atoms. Moreover, it is difficult to obtain the elastic properties of the heterogeneous nanostructured materials using atomistic simulations. For these and other reasons, it is prudent to seek a more practical approach. One such approach would be to extend the classical theory of elas- ticity down to the nanoscale by including in it the hitherto neglected surface/interface effect. For this it is necessary first to cast the latter within the framework of continuum elasticity.

Nano-mechanical resonators have attracted considerable attention recently due to their many important technological applications. Accurate analysis of various effects on the characteristics of resonators, such as resonant frequencies and quality factors, is crucial for designing high-performance components. Many authors have studied the vibration and heat transfer process of beams. Kidawa [4] has studied the problem of transverse vibrations of a beam induced by a mobile heat source. The analytical solution to the problem was obtained using the Green's functions method. However, Kidawa did not consider the thermoelastic coupling effect. Boley [5] analyzed the vibrations of a simply supported rectangular beam subjected to a suddenly applied heat input distributed along its span. Manolis and Beskos [6] examined the thermally induced vibration of structures consisting of beams, exposed to rapid surface heating. They have also studied the effects of damping and axial loads on the structural response. Al-Huniti et al. [7] investigated the thermally induced displacements and stresses of a rod using the Laplace transformation technique. Ai Kah Soh et al. studied the vibration of micro/nanoscale beam resonators induced by ultra-short-pulsed laser by considering the thermoelastic coupling term in $[8,9]$.

When very fast phenomena and small structure di- 
mensions are involved, the classical law of Fourier becomes inaccurate. A more sophisticated model is then needed to describe the thermal conduction mechanisms in a physically acceptable way. Modern technology has enabled the fabrication of materials and devices with characteristic dimensions of a few nanometers. Examples are super-lattices, nanowires, and quantum dots. At these length scales, the familiar continuum Fourier law for heat conduction is expected to fail due to both classical and quantum size effects [10]. Among many applications, the studying of the thermoelastic damping in MEMS /NEMS has been improved in $[11,13]$.

It is worthwhile to mention here that in most of the earlier studies, mechanical or thermal loading on the bounding surface is considered to be in the form of a shock. However, the sudden jump of the load is merely an idealized situation because it is impossible to realize a pulse described mathematically by a step function; even very rapid rise-time (of the order of $10^{-9} \mathrm{~s}$ ) may be slow in terms of the continuum. This is particularly true in the case of second sound effects when the thermal relaxation times for typical metals are less than $10^{-9} \mathrm{~s}$ [14]. It is thus felt that a finite time of rise of external load (mechanical or thermal) applied on the surface should be considered while studying a practical problem of this nature. Most ultrafast heat sources (such as certain lasers) involve the emission of a pulse (for example) that heats a material over a finite time due to the finite rise time of the pulse. Considering the aspect of rise of time, Misra et al. [15] and Youssef with many authors investigated many applications in which the ramp-type heating is used [1622].

In this paper, the non-Fourier effect in heat conduction, and the coupling effect between temperature and strain rate in nanoscale beam will be studied. In the present work, a generalized solution for the generalized thermoelastic vibration of a nano beam resonator induced by ramp type of heating will be developed. The solution takes into account the above two effects. The Laplace transformation method will be used to determine the lateral vibration, the temperature, the displacement, the stress and the energy of the beam. The effects of the relaxation time and the ramping time parameters will be studied and represented graphically.

\section{Problem Formulation}

Since beams with rectangular cross-sections are easy to fabricate, such cross-sections are commonly adopted in the design of NEMS resonators. Consider small flexural deflections of a thin elastic beam of length

$2 L(-L \leq x \leq L)$, width $b\left(-\frac{b}{2} \leq y \leq \frac{b}{2}\right)$ and thickness $h\left(-\frac{h}{2} \leq z \leq \frac{h}{2}\right)$, for which the $x, y$ and $z$ axes are de-

fined along the longitudinal, width and thickness directions of the beam, respectively. In equilibrium, the beam is unstrained, unstressed, and at temperature $T_{0}$ everywhere [8].

In the present study, the usual Euler-Bernoulli assumption [8,9] is adopted, i.e., any plane cross-section, initially perpendicular to the axis of the beam, remains plane and perpendicular to the neutral surface during bending. Thus, the displacements are given by

$$
u=-z \frac{\partial w(x, t)}{\partial x}, v=0, w(x, y, z, t)=w(x, t)
$$

Hence, the differential equation of thermally induced lateral vibration of the beam may be expressed in the form:

$$
\frac{\partial^{4} w}{\partial x^{4}}+\frac{\rho A}{E I} \frac{\partial^{2} w}{\partial t^{2}}+\alpha_{T} \frac{\partial^{2} M_{T}}{\partial x^{2}}=0,
$$

where $E$ is Young's modulus, $\mathrm{I}\left[=\mathrm{bh}^{3} / 12\right]$ is the inertial moment about x-axis, $\rho$ is the density of the beam, $\alpha_{T}$ is the coefficient of linear thermal expansion, $w(x, t)$ the lateral deflection, $\mathrm{x}$ is the distance along the length of the beam, $A=h b$ is the cross section area, $t$ is the time, and $M_{T}$ is the thermal moment, which is defined as

$$
M_{T}=\frac{12}{h^{3}} \int_{-h / 2}^{h / 2} \theta z \mathrm{~d} z,
$$

where $\theta=T-T_{0}$ is the dynamical temperature increment of the resonator, in which $T(x, z, t)$ is the temperature distribution and $T_{0}$ is the environmental temperature. The non-Fourier heat conduction equation has the following form [16-18]:

$$
\frac{\partial^{2} \theta}{\partial x^{2}}+\frac{\partial^{2} \theta}{\partial z^{2}}=\left(\frac{\partial}{\partial t}+\tau_{o} \frac{\partial^{2}}{\partial t^{2}}\right)\left(\frac{\rho C_{v}}{k} \theta+\frac{\beta T_{0}}{k} e\right),
$$

where $e=\frac{\partial u}{\partial x}+\frac{\partial v}{\partial y}+\frac{\partial w}{\partial z}$ is the volumetric strain, where $C_{v}$ is the specific heat at constant volume, $\tau_{o}$ is the thermal relaxation time, $k$ is the thermal conductivity and $\beta=\frac{E \alpha_{T}}{1-2 v}$ in which $v$ is Poisson's ratio.

Where there is no heat flow across the upper and lower surfaces of the beam, so that $\frac{\partial \theta}{\partial z}=0$ at $z= \pm h / 2$, for a very thin beam and assuming that the temperature varies in terms of a $\sin (p z)$ function along the thickness direction, where $p=\pi / h$, gives:

$$
\theta(x, z, t)=\theta_{1}(x, t) \sin (p z) .
$$


Hence, Equation (2) gives

$$
\frac{\partial^{4} w}{\partial x^{4}}+\frac{\rho A}{E I} \frac{\partial^{2} w}{\partial t^{2}}+\frac{12 \alpha_{T}}{h^{3}} \frac{\partial^{2} \theta_{1}}{\partial x^{2}} \int_{-h / 2}^{h / 2} z \sin (p z) \mathrm{d} z=0
$$

and Equation (4) gives

$$
\begin{aligned}
& \frac{\partial^{2} \theta_{1}}{\partial x^{2}} \sin (p z)-p^{2} \theta_{1} \sin (p z) \\
& =\left(\frac{\partial}{\partial t}+\tau_{o} \frac{\partial^{2}}{\partial t^{2}}\right)\left(\frac{\rho C_{v}}{k} \theta_{1} \sin (p z)-\frac{\beta T_{0}}{k} z \frac{\partial^{2} w}{\partial x^{2}}\right)
\end{aligned}
$$
form

After doing the integrations, Equation (5) takes the

$$
\frac{\partial^{4} w}{\partial x^{4}}+\frac{\rho A}{E I} \frac{\partial^{2} w}{\partial t^{2}}+\frac{24 \alpha_{T}}{h \pi^{2}} \frac{\partial^{2} \theta_{1}}{\partial x^{2}}=0 .
$$

In Equation (6), we multiply the both sides by $\mathrm{z}$ and integrating with respect to $z$ from $-h / 2$ to $h / 2$, then we obtain

$$
\left(\frac{\partial^{2} \theta_{1}}{\partial x^{2}}-p^{2} \theta_{1}\right)=\left(\frac{\partial}{\partial t}+\tau_{o} \frac{\partial^{2}}{\partial t^{2}}\right)\left(\eta \theta_{1}-\frac{\beta T_{0} \pi^{2} h}{24 k} \frac{\partial^{2} w}{\partial x^{2}}\right),
$$

where $\eta=\frac{\rho C_{v}}{k}$.

Now, for simplicity we will use the following nondimensional variables:

$$
\begin{aligned}
& \left(x^{\prime}, w^{\prime}, h^{\prime}\right)=\eta c_{o}(x, w, h),\left(t^{\prime}, \tau_{o}^{\prime}\right)=\eta c_{o}^{2}\left(t, \tau_{o}\right), \\
& \sigma^{\prime}=\frac{\sigma}{E}, \theta_{1}^{\prime}=\frac{\theta_{1}}{T_{o}}, c_{o}^{2}=\frac{E}{\rho}
\end{aligned}
$$

Then, we have

$$
\frac{\partial^{4} w}{\partial x^{4}}+A_{1} \frac{\partial^{2} w}{\partial t^{2}}+A_{2} \frac{\partial^{2} \theta_{1}}{\partial x^{2}}=0
$$

and

$$
\frac{\partial^{2} \theta_{1}}{\partial x^{2}}-A_{3} \theta_{1}=\left(\frac{\partial}{\partial t}+\tau_{o} \frac{\partial^{2}}{\partial t^{2}}\right)\left(\theta_{1}-A_{4} \frac{\partial^{2} w}{\partial x^{2}}\right),
$$

where

$$
A_{1}=\frac{12}{h^{2}}, A_{2}=\frac{24 \alpha_{t} T_{o}}{\pi^{2} h}, A_{3}=p^{2}, A_{4}=\frac{\pi^{2} \beta h}{24 k \eta},
$$

and we have canceled the prime for convenient.

\section{Formulations the Problem in the Laplace Transform Domain}

Applying the Laplace transform for Equations (10) and (11) defined by the formula

$$
\bar{f}(s)=L[f(t)]=\int_{0}^{\infty} f(t) \mathrm{e}^{-s t} \mathrm{~d} t .
$$

Hence, we obtain the following system of differential equations

$$
\frac{\mathrm{d}^{4} \bar{w}}{\mathrm{~d} x^{4}}+A_{1} s^{2} \bar{w}+A_{2} \frac{\mathrm{d}^{2} \overline{\theta_{1}}}{\mathrm{~d} x^{2}}=0,
$$

and

$$
\frac{\mathrm{d}^{2} \bar{\theta}_{1}}{\mathrm{~d} x^{2}}-A_{3} \bar{\theta}_{1}=\left(s+\tau_{o} s^{2}\right)\left(\bar{\theta}_{1}-A_{4} \frac{\mathrm{d}^{2} \bar{w}}{\mathrm{~d} x^{2}}\right) .
$$

We have considered all the initial states of variables were zero, i.e.

$$
\theta_{1}(x, 0)=\frac{\mathrm{d} \theta_{1}(x, 0)}{\mathrm{d} x}=w(x, 0)=\frac{\mathrm{d} w(x, 0)}{\mathrm{d} x}=0
$$

We can re-write the above system of equations in the form

$$
\left(\frac{\mathrm{d}^{4}}{\mathrm{~d} x^{4}}+A_{1} s^{2}\right) \bar{w}+A_{2} \frac{\mathrm{d}^{2} \overline{\theta_{1}}}{\mathrm{~d} x^{2}}=0,
$$

and

$$
\left(\frac{\mathrm{d}^{2}}{\mathrm{~d} x^{2}}-\alpha_{1}\right) \bar{\theta}_{1}=-\alpha_{2} \frac{\mathrm{d}^{2} \bar{w}}{\mathrm{~d} x^{2}}
$$

where

$$
\alpha_{1}=A_{3}+\left(s+\tau_{o} s^{2}\right), \alpha_{2}=\left(s+\tau_{o} s^{2}\right) A_{4}
$$

By eliminating $\bar{w}$ from the above system of equation, we get

$$
\left[D^{6}-\left(\alpha_{1}+\alpha_{2} A_{2}\right) D^{4}+A_{1} s^{2} D^{2}-A_{1} \alpha_{1} s^{2}\right] \bar{w}=0,
$$

where $\bar{\theta}_{1}$ satisfies the same equation, i.e.

$$
\left[D^{6}-\left(\alpha_{1}+\alpha_{2} A_{2}\right) D^{4}+A_{1} s^{2} D^{2}-A_{1} \alpha_{1} s^{2}\right] \bar{\theta}_{1}=0 \text {. }
$$

The characteristic equation can be presented as

$$
\lambda^{6}-\ell \lambda^{4}+m \lambda^{2}-n=0
$$

The roots of this equation, namely, $\pm \lambda_{1}, \pm \lambda_{2}$ and $\pm \lambda_{3}$, satisfy the following relations

$$
\begin{gathered}
\lambda_{1}^{2}+\lambda_{2}^{2}+\lambda_{3}^{2}=\alpha_{1}+\alpha_{2} A_{2}=\ell, \\
\lambda_{1}^{2} \lambda_{2}^{2}+\lambda_{2}^{2} \lambda_{3}^{2}+\lambda_{1}^{2} \lambda_{3}^{2}=A_{1} s^{2}=m, \\
\lambda_{1}^{2}+\lambda_{2}^{2}+\lambda_{3}^{2}=\alpha_{1} A_{1} s^{2}=n .
\end{gathered}
$$

We can consider the solution take the form

$$
\bar{w}=\sum_{i=1}^{6} C_{i} \exp \left(\lambda_{i} x\right),
$$

and

$$
\overline{\theta_{1}}=\sum_{i=1}^{6} E_{i} \exp \left(\lambda_{i} x\right),
$$


where $C_{i}$ and $E_{i}(i=1,2, \cdots, 6)$ are some parameters depend, only, on $s$.

Using Equation (16) and (21), we get

$$
E_{i}=-\frac{\alpha_{2} \lambda_{i}^{2} C_{i}}{\left(\lambda_{i}^{2}-\alpha_{1}\right)},
$$

this gives

$$
\overline{\theta_{1}}=-\alpha_{2} \sum_{i=1}^{6} \frac{\lambda_{i}^{2}}{\left(\lambda_{i}^{2}-\alpha_{1}\right)} C_{i} \exp \left(\lambda_{i} x\right) .
$$

Now, to get the values of the constants $C_{i}$ and $E_{i}$ we will consider the two ends of the micro-beams are clamped, then the boundary conditions are $[8,9]$ :

$$
w( \pm L, t)=\frac{\mathrm{d}^{2} w( \pm L, t)}{\mathrm{d} x^{2}}=0,
$$

and loaded thermally by ramp-type heating, which give

$$
\theta_{1}( \pm L, t)=\theta_{0}\left[\begin{array}{ccc}
0 & \text { for } & t \leq 0 \\
\frac{t}{t_{0}} & \text { for } & 0<t<t_{0} \\
1 & \text { for } & t \geq t_{0}
\end{array}\right] \text {, }
$$

where $t_{0}$ is non-negative constant and is called ramp- type parameter and $\theta_{0}$ is constant [18].

After using Laplace transform, the above conditions take the forms

$$
\bar{w}( \pm L, s)=\frac{\mathrm{d}^{2} \bar{w}( \pm L, s)}{\mathrm{d} x^{2}}=0,
$$

and

$$
\bar{\theta}_{1}(s)=\frac{\theta_{0}}{t_{0}}\left(\frac{1-e^{-t_{0} s}}{s^{2}}\right)=F(s) .
$$

Applying the conditions (25) and (26) into Equations (20) and (22), we obtain

$$
\begin{gathered}
\sum_{i=1}^{3} C_{i} \cosh \left(\lambda_{i} L\right)=0, \\
\sum_{i=1}^{3} \lambda_{i}^{2} C_{i} \cosh \left(\lambda_{i} L\right)=0,
\end{gathered}
$$

$$
\sum_{i=1}^{3} \frac{\lambda_{i}^{2}}{\left(\lambda_{i}^{2}-\alpha_{1}\right)} C_{i} \cosh \left(\lambda_{i} L\right)=-\frac{F(s)}{\alpha_{2}} .
$$

By solving the above system of linear algebraically equations, we get

The lateral deflection

$$
\bar{w}(x, s)=G\left[\frac{\cosh \left(\lambda_{1} x\right) / \cosh \left(\lambda_{1} L\right)}{\left(\lambda_{1}^{2}-\lambda_{2}^{2}\right)\left(\lambda_{1}^{2}-\lambda_{3}^{2}\right)}+\frac{\cosh \left(\lambda_{2} x\right) / \cosh \left(\lambda_{2} L\right)}{\left(\lambda_{1}^{2}-\lambda_{2}^{2}\right)\left(\lambda_{3}^{2}-\lambda_{2}^{2}\right)}+\frac{\cosh \left(\lambda_{3} x\right) / \cosh \left(\lambda_{3} L\right)}{\left(\lambda_{1}^{2}-\lambda_{3}^{2}\right)\left(\lambda_{2}^{2}-\lambda_{3}^{2}\right)}\right]
$$

The temperature

$$
\begin{aligned}
\bar{\theta}(x, z, s)= & -\alpha_{2} G \sin (p z)\left[\frac{\lambda_{1}^{2} \cosh \left(\lambda_{1} x\right) / \cosh \left(\lambda_{1} L\right)}{\left(\lambda_{1}^{2}-\alpha_{1}\right)\left(\lambda_{1}^{2}-\lambda_{2}^{2}\right)\left(\lambda_{1}^{2}-\lambda_{3}^{2}\right)}+\frac{\lambda_{2}^{2} \cosh \left(\lambda_{2} x\right) / \cosh \left(\lambda_{2} L\right)}{\left(\lambda_{2}^{2}-\alpha_{1}\right)\left(\lambda_{1}^{2}-\lambda_{2}^{2}\right)\left(\lambda_{3}^{2}-\lambda_{2}^{2}\right)},\right. \\
& \left.+\frac{\lambda_{3}^{2} \cosh \left(\lambda_{3} x\right) / \cosh \left(\lambda_{3} L\right)}{\left(\lambda_{3}^{2}-\alpha_{1}\right)\left(\lambda_{1}^{2}-\lambda_{3}^{2}\right)\left(\lambda_{2}^{2}-\lambda_{3}^{2}\right)}\right]
\end{aligned}
$$

The displacement

$$
\bar{u}(x, z, s)=-z G\left[\frac{\lambda_{1} \sinh \left(\lambda_{1} x\right) / \cosh \left(\lambda_{1} L\right)}{\left(\lambda_{1}^{2}-\lambda_{2}^{2}\right)\left(\lambda_{1}^{2}-\lambda_{3}^{2}\right)}+\frac{\lambda_{2} \sinh \left(\lambda_{2} x\right) / \cosh \left(\lambda_{2} L\right)}{\left(\lambda_{1}^{2}-\lambda_{2}^{2}\right)\left(\lambda_{3}^{2}-\lambda_{2}^{2}\right)}+\frac{\lambda_{3} \sinh \left(\lambda_{3} x\right) / \cosh \left(\lambda_{3} L\right)}{\left(\lambda_{1}^{2}-\lambda_{3}^{2}\right)\left(\lambda_{2}^{2}-\lambda_{3}^{2}\right)}\right],
$$

where

$$
G=\frac{F(s)\left(\alpha_{1}-\lambda_{1}^{2}\right)\left(\alpha_{1}-\lambda_{2}^{2}\right)\left(\alpha_{1}-\lambda_{3}^{2}\right)}{\alpha_{1} \alpha_{2}}
$$

$$
\sigma_{x x}(x, z, t)=E\left(\frac{\partial u}{\partial x}-\alpha_{T} \theta\right),
$$

By using the non-dimensional variables in (9), we obtain the stress in the form

$$
\sigma_{x x}(x, z, t)=\frac{\partial u}{\partial x}-\alpha_{T} T_{0} \theta .
$$

\section{The Stress and the Strain Energy}

The stress on the x-axis, according to Hooke's law is

After using Laplace transform, the above equation gives 


$$
\begin{aligned}
& \bar{\sigma}_{x x}(x, z, s)=\frac{\partial \bar{u}}{\partial x}-\alpha_{T} T_{0} \bar{\theta} . \\
& \bar{\sigma}_{x x}(x, z, s)=-z G\left[\frac{\lambda_{1}^{2} \cosh \left(\lambda_{1} x\right) / \cosh \left(\lambda_{1} L\right)}{\left(\lambda_{1}^{2}-\lambda_{2}^{2}\right)\left(\lambda_{1}^{2}-\lambda_{3}^{2}\right)}+\frac{\lambda_{2}^{2} \cosh \left(\lambda_{2} x\right) / \cosh \left(\lambda_{2} L\right)}{\left(\lambda_{1}^{2}-\lambda_{2}^{2}\right)\left(\lambda_{3}^{2}-\lambda_{2}^{2}\right)}\right. \\
& \left.+\frac{\lambda_{3}^{2} \cosh \left(\lambda_{3} x\right) / \cosh \left(\lambda_{3} L\right)}{\left(\lambda_{1}^{2}-\lambda_{3}^{2}\right)\left(\lambda_{2}^{2}-\lambda_{3}^{2}\right)}\right] \\
& +\alpha_{T} T_{0} \alpha_{2} G \sin (p z)\left[\frac{\lambda_{1}^{2} \cosh \left(\lambda_{1} x\right) / \cosh \left(\lambda_{1} L\right)}{\left(\lambda_{1}^{2}-\alpha_{1}\right)\left(\lambda_{1}^{2}-\lambda_{2}^{2}\right)\left(\lambda_{1}^{2}-\lambda_{3}^{2}\right)}+\frac{\lambda_{2}^{2} \cosh \left(\lambda_{2} x\right) / \cosh \left(\lambda_{2} L\right)}{\left(\lambda_{2}^{2}-\alpha_{1}\right)\left(\lambda_{1}^{2}-\lambda_{2}^{2}\right)\left(\lambda_{3}^{2}-\lambda_{2}^{2}\right)}\right. \\
& \left.+\frac{\lambda_{3}^{2} \cosh \left(\lambda_{3} x\right) / \cosh \left(\lambda_{3} L\right)}{\left(\lambda_{3}^{2}-\alpha_{1}\right)\left(\lambda_{1}^{2}-\lambda_{3}^{2}\right)\left(\lambda_{2}^{2}-\lambda_{3}^{2}\right)}\right]
\end{aligned}
$$

By using Equations (31) and (32) with equation (35), by

The energy which is generated on the beam is given

$$
W=\sum_{i, j=1}^{3} \frac{1}{2} \sigma_{i j} e_{i j}=\frac{1}{2} \sigma_{x x} e_{x x}=-\frac{1}{2} z \sigma_{x x} \frac{\partial^{2} w}{\partial x^{2}},
$$

or, we can write as follows:

$$
W=-\frac{1}{2} z\left[L^{-1}\left(\bar{\sigma}_{x x}\right)\right]\left[L^{-1}\left(\frac{\partial^{2} \bar{w}}{\partial x^{2}}\right)\right],
$$

where $L^{-1}[\bar{f}(s)]=f(t)$ is the Laplace inverse transform.

\section{Numerical Inversion of the Laplace Transform}

In order to determine the solutions in the time domain, the Riemann-sum approximation method is used to obtain the numerical results. In this method, any function in Laplace domain can be inverted to the time domain as

$$
f(t)=\frac{e^{\kappa t}}{t}\left[\frac{1}{2} \bar{f}(\kappa)+\operatorname{Re} \sum_{n=1}^{N}(-1)^{n} \bar{f}\left(\kappa+\frac{i n \pi}{t}\right)\right]
$$

where Re is the real part and $i$ is imaginary number unit. For faster convergence, numerous numerical experiments have shown that the value of $\kappa$ satisfies the relation $\kappa t \approx 4.7 \quad$ [23].

\section{Numerical Results and Discussion}

Now, we will consider a numerical example for which computational results are given. For this purpose, Silicon is taken as the thermoelastic material for which we take the following values of the different physical constants [24]:

$$
\begin{gathered}
K=156 \mathrm{~W} /(\mathrm{mK}), \quad \alpha_{T}=2.59(10)^{-6} \mathrm{k}^{-1}, \\
\rho=2330 \mathrm{~kg} / \mathrm{m}^{3}, T_{0}=293 \mathrm{k}, \\
C_{v}=713 \mathrm{~J} /(\mathrm{kgK}) E=169 \mathrm{GPa}, \quad v=0.22 .
\end{gathered}
$$

The aspect ratios of the beam are fixed as $L / h=10$ and $b / h=1 / 2$, when $h$ is varied, $L$ and $b$ change accordingly with $h$.

For the nanoscale beam, we will take the range of the beam length $L(1-100) \times 10^{-12} \mathrm{~m}$. The original time $t$ and the ramping time parameter $t_{0}$ will be considered in the picoseconds $(1-100) \times 10^{-12}$ sec and the relaxation time $\tau_{0}$ in the range $(1-100) \times 10^{-14} \mathrm{sec}$.

The figures were prepared by using the non-dimensional variables which are defined in (9) for a wide range of beam length $2 L$ when $L=1.0, \theta_{0}=1.0 \quad z=h / 6$ and $t=0.15$.

In Figures 1-5, we represented the lateral vibration, the temperature, the displacement, the stress and the energy of the beam at different values of the relaxation time when $\tau_{0}=0.0$ (Biot model) and $\tau_{0}=0.02$ (L-S model) and we have found that, the relaxation time has significant effects on all the studied fields. In the context of L-S model, the relaxation time gives values of the lateral vibration, the temperature, the displacement, the stress and the energy less than their values in the context of Biot model which is very obvious in the peek points.

We can say that, in the context of the generalized thermoelasticity the speed of the wave propagation of all the studied fields are finite and the damping of the strain energy increasing.

In Figures 6-10, we represented the lateral vibration, the temperature, the displacement, the stress and the energy of the beam at different values of the ramping time parameter when $t_{0}(=0.10)<t(=0.15)$, $t_{0}=t=0.15$ and $t_{0}(=0.20)>t(=0.15)$ in the context 


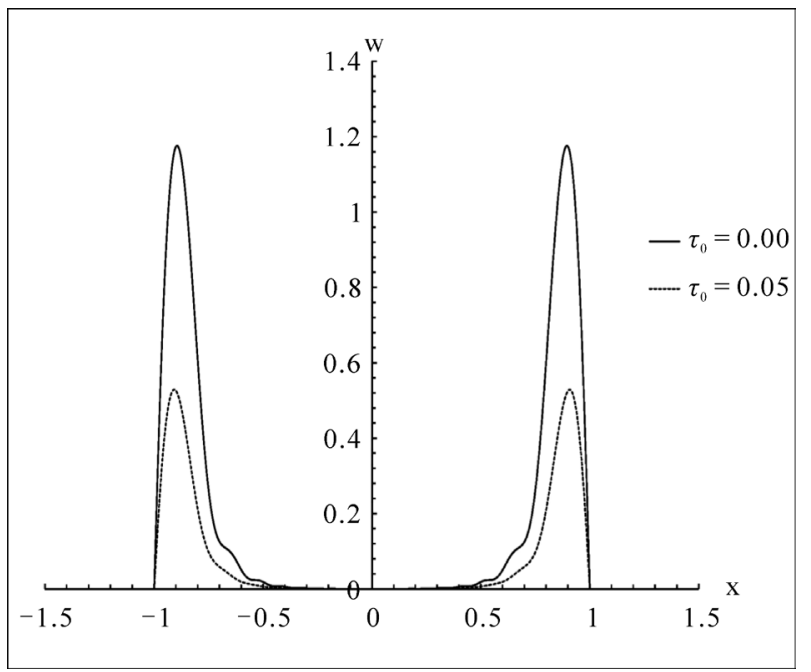

Figure 1. The lateral deflection w for L-S and Biot theories.

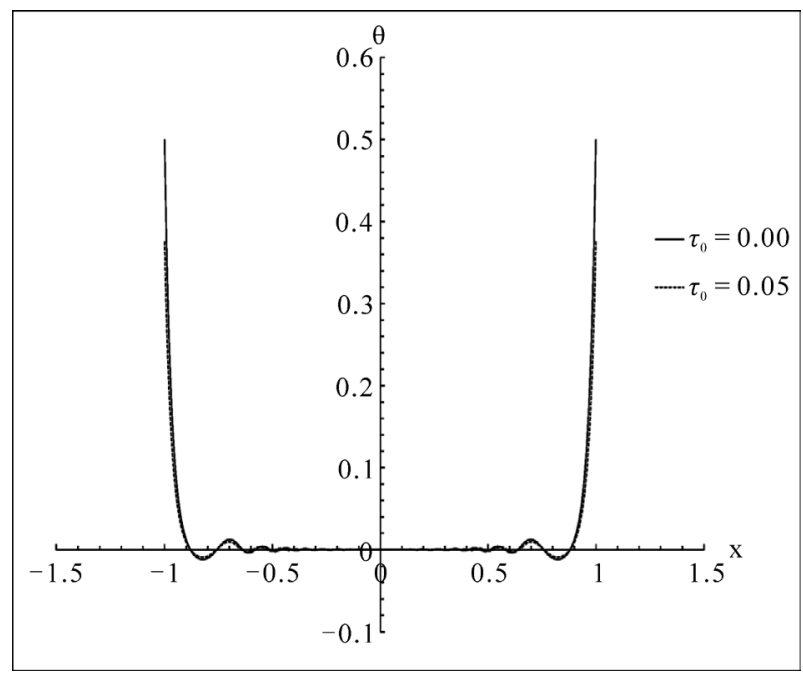

Figure 2. The temperature for L-S and Biot theories.

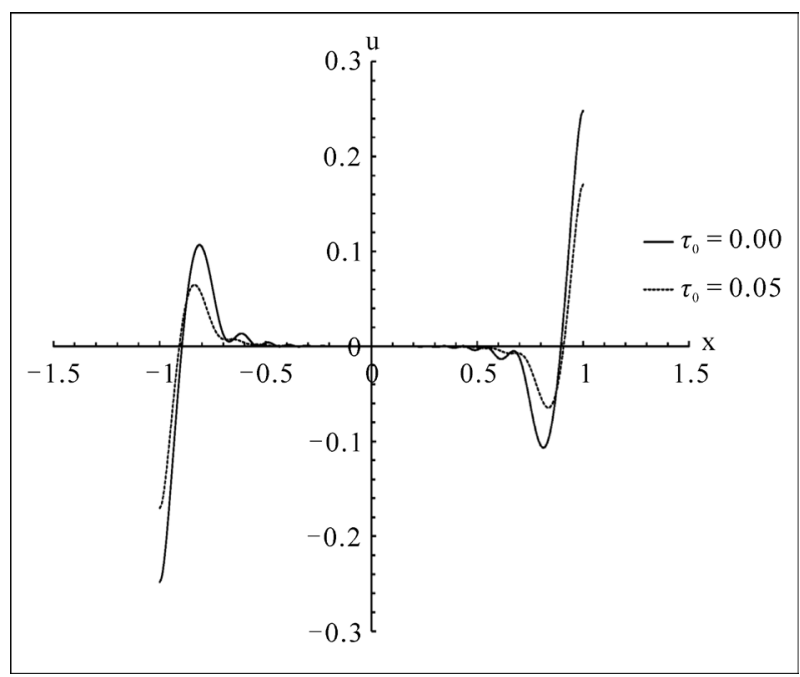

Figure 3. The displacement for L-S and Biot theories.

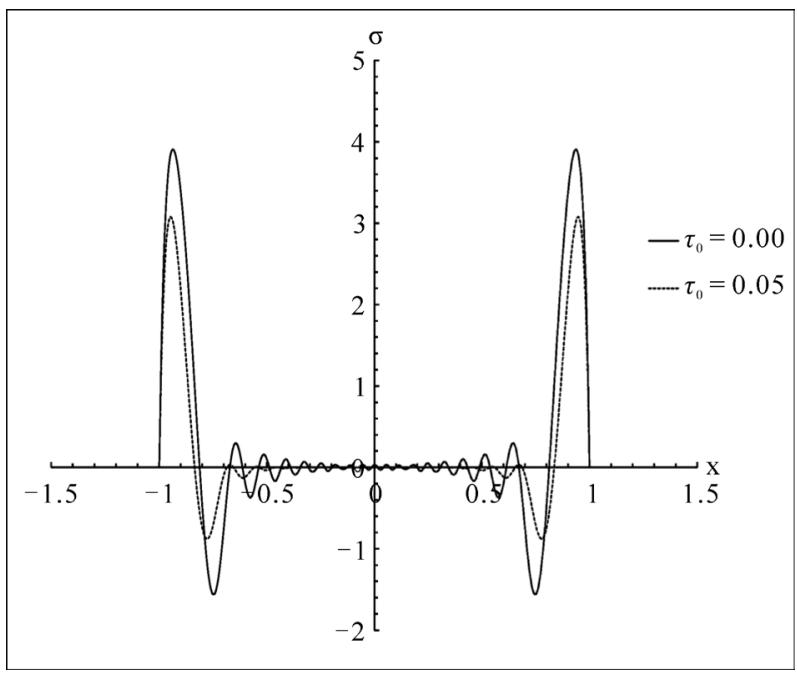

Figure 4. The stress for $L-S$ and Biot theories.

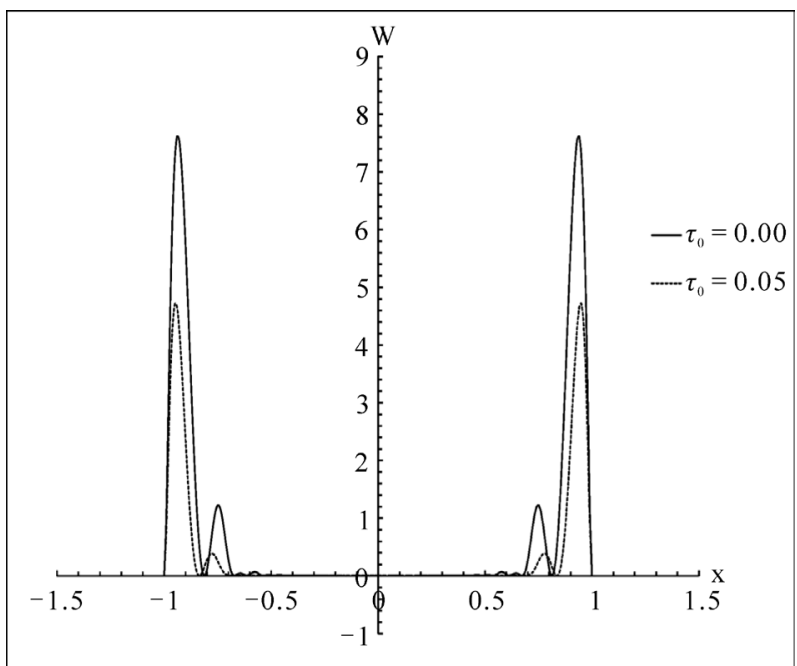

Figure 5. The energy for L-S and Biot theories.

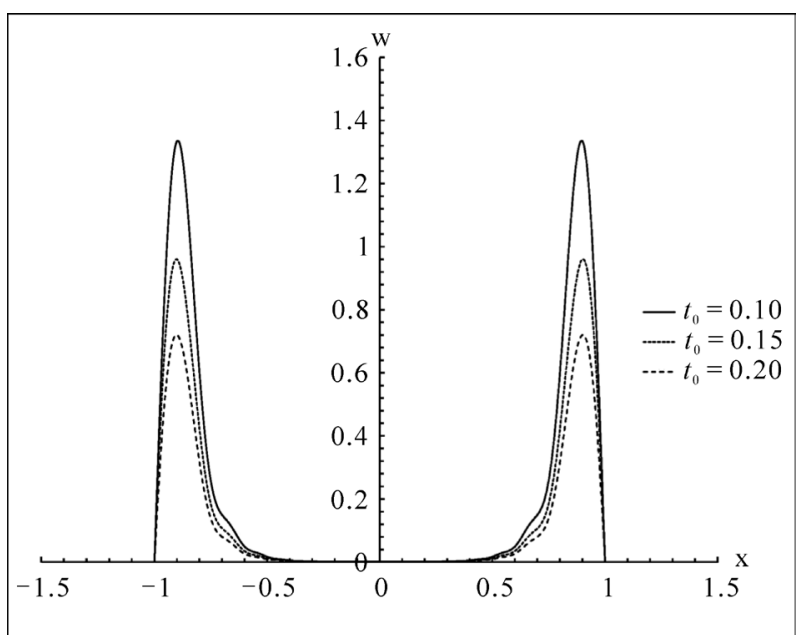

Figure 6. The lateral deflection $w$ at different time of ramping parameter. 


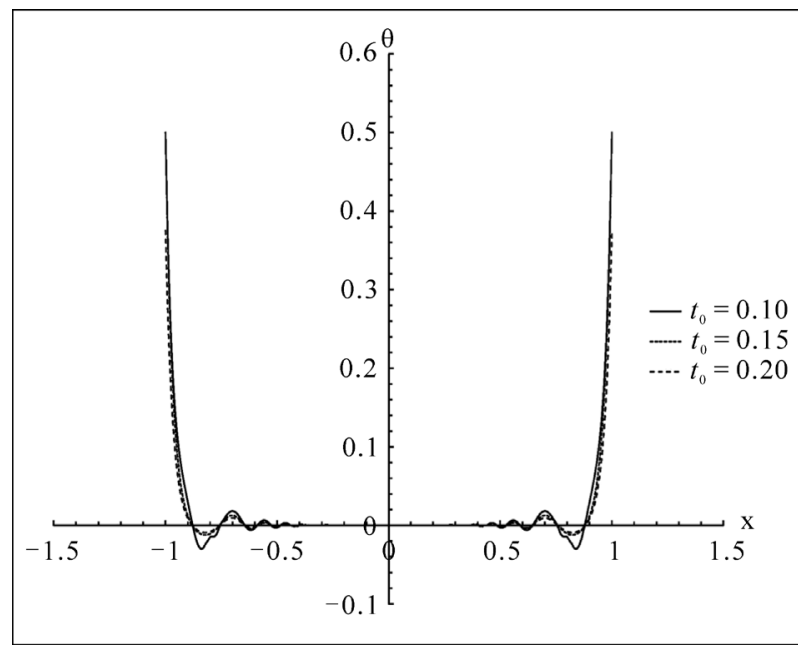

Figure 7. The temperature at different time of ramping parameter.

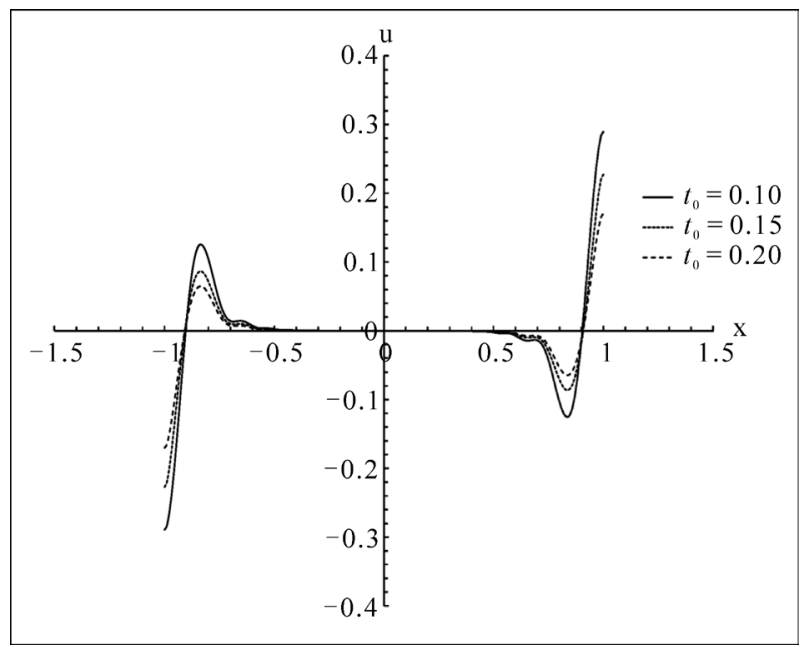

Figure 8. The displacement at different time of ramping parameter.

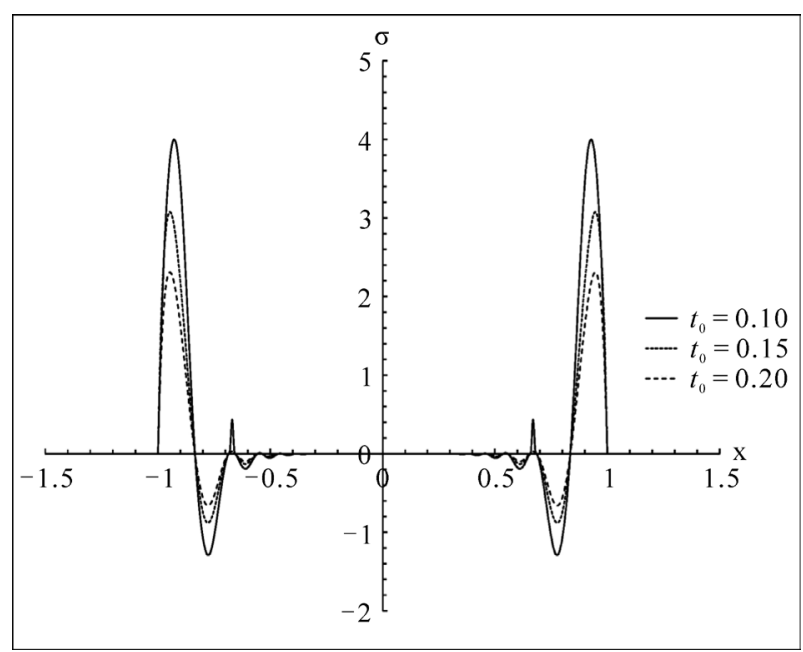

Figure 9. The stress at different time of ramping parameter.

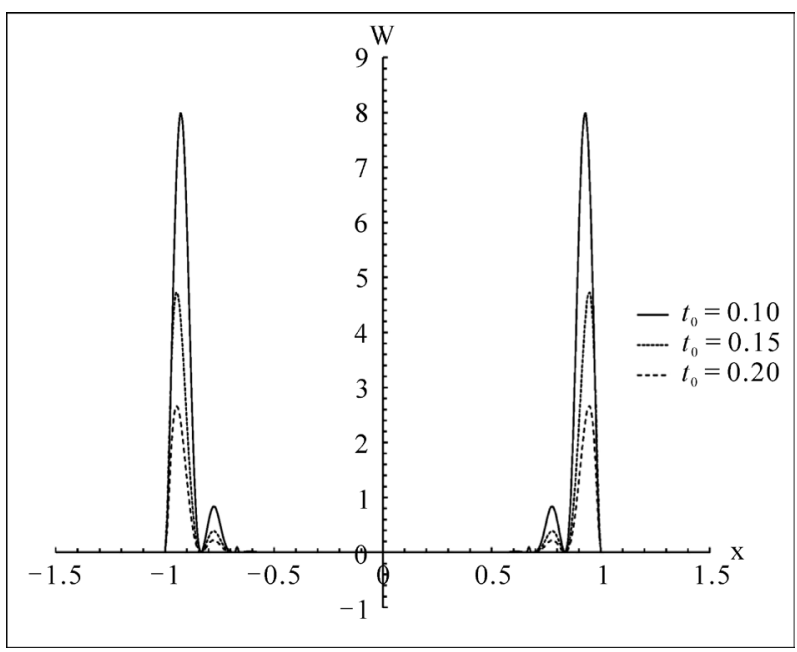

Figure 10. The energy at different time of ramping parameter.

of L-S model.

We have found that, the ramping time parameter has significant effects on all the studied fields. The increasing in the value of the ramping time parameter causes decreasing in the values of the lateral vibration, the temperature, the displacement, the stress and the energy which are very obvious in the peek points of the curves. Also, the damping of the strain energy is increases when the ramping time parameter increases.

\section{Conclusions}

This paper has investigated the vibration characteristics of the deflection, the temperature, the displacement, the stress and the strain energy of an Euler-Bernoulli beam induced by a ramp type heating. An analytical direct method and numerical technique based on the Laplace transformation has been used to calculate the vibration of the deflection, the temperature, the displacement, the stress and the strain energy. The effects of the relaxation time and the ramping time parameter on all the studied fields have been shown and represented graphically. The non-Fourier law of heat conduction gives a finite speed of wave propagation and increases the damping of the strain energy.

\section{References}

[1] M. Biot, "Thermoelasticity and Irreversible ThermoDynamics,” Journal of Applied Physics, Vol. 27, No. , 1956, pp. 240-253. doi:10.1063/1.1722351

[2] H. Lord and Y. Shulman, "A Generalized Dynamical Theory of Thermoelasticity," Journal of Mechanics and Physics of Solids, Vol. 15, No. 5, September 1967, pp. 299-309. doi:10.1016/0022-5096(67)90024-5 
[3] J. K. Diao, K. Gall and M. L. Dunn, “Atomistic Simulation of the Structure and Elastic Properties of Gold Nanowires," Journal of Mechanics and Physics of Solids, Vol. 52, No. 9, September 2004, pp.1935-1962. doi:10.1016/j.jmps.2004.03.009

[4] J. Kidawa-Kukla, “Application of the Green Functions to the Problem of the Thermally Induced Vibration of a Beam,” Journal of Sound and Vibration, Vol. 262, No. 4, May 2003, pp. 865-876. doi:10.1016/S0022-460X(02)01133-1

[5] B. A. Boley, "Approximate Analyses of Thermally Induced Vibrations of Beams and Plates," Journal of Applied Mechanics, Vol. 39, No. 1, 1972, pp. 212-216. doi:10.1115/1.3422615

[6] G. D. Manolis and D. E. Beskos, “Thermally Induced Vibrations of Beam Structures," Computer Methods in Applied Mechanics and Engineering, Vol. 21, No. 3, March 1980, pp. 337-355. doi:10.1016/0045-7825(80)90101-2

[7] N. S. Al-Huniti, M. A. Al-Nimr and M. Naij, "Dynamic Response of a Rod Due to a Moving Heat Source under the Hyperbolic Heat Conduction Model," Journal of Sound and Vibration, Vol. 242, No. 4, May 2001, pp. 629-640. doi:10.1006/jsvi.2000.3383

[8] A. K. Soh, Y. X. Sun and D. N. Fang, "Vibration of Microscale Beam Induced by Laser Pulse," Journal of Sound and Vibration, Vol. 311, No. 1-2, March 2008, pp. 243-253. doi:10.1016/j.jsv.2007.09.002

[9] Y. X. Sun, D. N. Fang, M. Saka and A. K. Soh, "Laser-Induced Vibrations of Micro-Beams under Different Boundary Conditions," International Journal of Solids and Structures, Vol. 45, No. 7-8, April 2008, pp. 19932013. doi:10.1016/j.ijsolstr.2007.11.006

[10] J. S. Rao, "Nonlinear Vibration and One Dimensional Structures," Advanced Theory of Vibration, Wiley, New York, 1992.

[11] Y. X. Sun, D. N. Fang and A. K. Soh, "Thermoelastic Damping in Micro-Beam Resonators," International Journal of Solids and Structures, Vol. 43, No. 10, May 2006, pp. 3213-3229. doi:10.1016/j.ijsolstr.2005.08.011

[12] D. N. Fang, Y. X. Sun and A. K. Soh, “Analysis of Frequency Spectrum of Laser-Induced Vibration of Microbeam Resonators," Chinese Physics Letters, Vol. 23, No. 6, 2006, pp. 1554-1557.

[13] A. Duwel, J. Gorman, M. Weinstein, J. Borenstein and P. Ward, "Experimental Study of Thermoelastic Damping in MEMS Gyros," Sensors and Actuators A, Vol. 103, No. 1-2, January 2003, pp. 70-75. doi:10.1016/S0924-4247(02)00318-7

[14] J. C. Misra, S. C. Samanta, A. K. Chakrabarti and S. C.
Misra, "Magnetothermoelastic Interaction in an Infinite Elastic Continuum with a Cylindrical Hole Subjected to Ramp-Type Heating”, International Journal of Engineering Science, Vol. 29, No. 12, 1991, pp. 1505-1514. doi:10.1016/0020-7225(91)90122-J

[15] J. C. Misra, S. C. Samanta and A. K. Chakrabarti, "Magnetothermoelastic Interaction in an Aeolotropic Solid Cylinder Subjected to a Ramp-Type Heating”, International Journal of Engineering Science, Vol. 29, No. 9, 1991, pp. 1065-1075. doi:10.1016/0020-7225(91)90112-G

[16] H. M. Youssef, "State-Space on Generalized Thermoelasticity for an Infinite Material with a Spherical Cavity and Variable Thermal conductivity Subjected to RampType Heating," Journal of CAMQ, Applied Mathematics Institute, Vol. 13, No. 4, 2005, pp. 369-390.

[17] H. M. Youssef, "Problem of Generalized Thermoelastic Infinite Medium with Cylindrical Cavity Subjected to a Ramp-Type Heating and Loading," Journal of Archive of Applied Mechanics, Vol. 75, No. , 2006, pp. 553-565. doi:10.1007/s00419-005-0440-3

[18] H. M. Youssef, “Two-Dimensional Generalized Thermoelasticity Problem for a Half-Space Subjected to Ramp-Type Heating,” European Journal of MechanicsA/Solids, Vol. 25, No. 5, September-October 2006, pp. 745-763. doi:10.1016/j.euromechsol.2005.11.005

[19] H. M. Youssef and A. H. Al-Harby, "State-Space Approach of Two-Temperature Generalized Thermoelasticity of Infinite Body with a Spherical Cavity Subjected to Different Types of Thermal Loading," Journal of Archive of Applied Mechanics, Vol. 77, No. 9, 2007, pp. 675-687. doi:10.1007/s00419-007-0120-6

[20] H. M. Youssef, "Two-Dimensional Problem of TwoTemperature Generalized Thermoelastic Half-Space Subjected to Ramp-Type Heating," Journal of Computational Mathematics and Modeling, Vol.19, No. 2, 2008, pp. 201-235. doi:10.1007/s10598-008-0014-7

[21] M. A. Ezzat and H. M. Youssef, "Stat-Space Approach of Conducting Magneto-Thermoelastic Medium With Variable Thermal Conductivity Subjected To Ramp-Type Heating," Journal of Thermal Stresses, Vol. 32, No. , 2009, pp. 414-427. doi:10.1080/01495730802637233

[22] H. M. Youssef and A.A. El-Bary, "Generalized Thermoelastic Infinite Layer Subjected to Ramp-Type Thermal and Mechanical Loading under Three Theories-State Space Approach,” Journal of Thermal Stresses, Vol. 32, No. , 2009, pp. 1-18.

[23] D. Tzou, “Macro-to-micro heat transfer,” Taylor \& Francis, Washington DC, 1996.

[24] R. Hull, "Properties of Silicon, INSPEC," The Institution of Electrical Engineers, London, New York, 1988. 